Marquette University

e-Publications@Marquette

\title{
Multiple States of Nitrile Hydratase from Rhodococcus equi TG328-2: Structural and Mechanistic Insights from Electron Paramagnetic Resonance and Density Functional Theory Studies
}

\author{
Natalia Stein \\ University of Wisconsin - Milwaukee \\ Natalie Gumataotao \\ Loyola University Chicago
}

Natalia Hajnas

Loyola University Chicago

Rui Wu

Loyola University Chicago

Karunagala Pathiranage Wasantha Lankathilaka

Marquette University, wasanthalankathilaka.karunagalapathiranage@marquette.edu

See next page for additional authors

Follow this and additional works at: https://epublications.marquette.edu/physics_fac

Part of the Physics Commons

\section{Recommended Citation}

Stein, Natalia; Gumataotao, Natalie; Hajnas, Natalia; Wu, Rui; Wasantha Lankathilaka, Karunagala Pathiranage; Bornscheuer, Uwe; Liu, Dali; Fiedler, Adam T.; Holz, Richard C.; and Bennett, Brian, "Multiple States of Nitrile Hydratase from Rhodococcus equi TG328-2: Structural and Mechanistic Insights from Electron Paramagnetic Resonance and Density Functional Theory Studies" (2017). Physics Faculty Research and Publications. 144.

https://epublications.marquette.edu/physics_fac/144 


\section{Authors}

Natalia Stein, Natalie Gumataotao, Natalia Hajnas, Rui Wu, Karunagala Pathiranage Wasantha Lankathilaka, Uwe Bornscheuer, Dali Liu, Adam T. Fiedler, Richard C. Holz, and Brian Bennett 


\section{Marquette University \\ e-Publications@Marquette}

\section{Physics Faculty Research and Publications/College of Arts and Sciences}

This paper is NOT THE PUBLISHED VERSION; but the author's final, peer-reviewed manuscript. The published version may be accessed by following the link in the citation below.

Biochemistry, Vol. 56, No. 24, (2017): 3068-3077. DOI. This article is (C) American Chemical Society and permission has been granted for this version to appear in $\underline{\mathrm{e}-}$ Publications@Marquette. American Chemical Society does not grant permission for this article to be further copied/distributed or hosted elsewhere without the express permission from American Chemical Society.

\section{Contents}

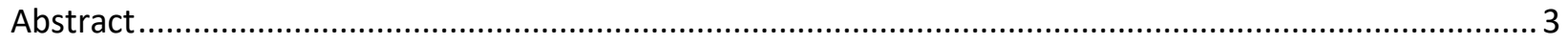

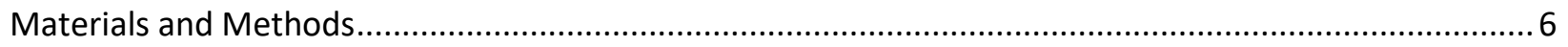

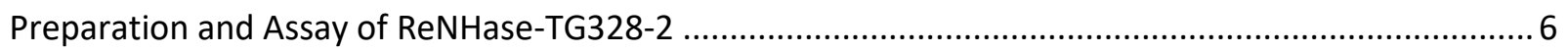

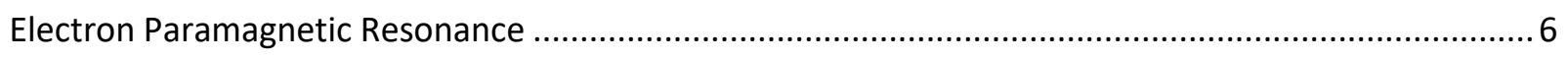

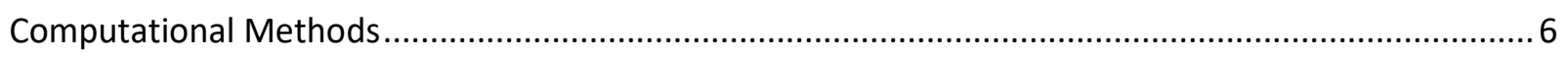

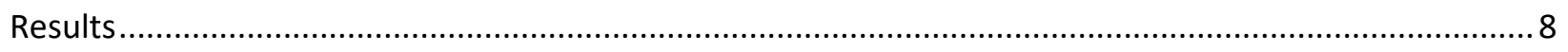

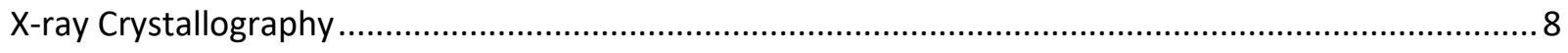

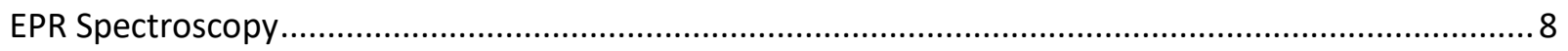

ReNHase-TG328-2 Prepared Aerobically in the Presence of Butyric Acid ........................................... 8

Anaerobic Preparation, Air Oxidation, and Butyric Acid Exposure of ReNHase-TG328-2 .................... 10

ReNHase-TG328-2 upon Incubation with Alkylboronate Inhibitors ................................................ 11

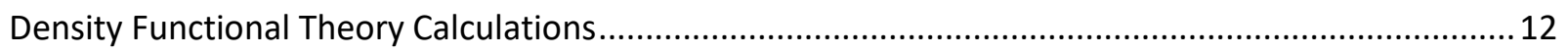

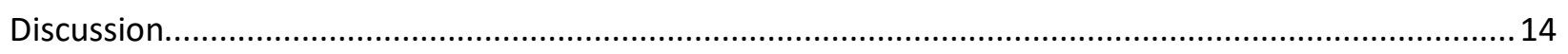

Origins of Multiple Chemical Species in As-Prepared ReNHase-TG328-2 …..................................... 14

EPR of ReNHase-TG328-2 upon Incubation with Alkylboronate Inhibitors ...................................... 17

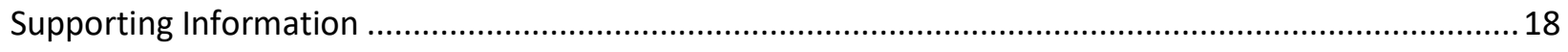

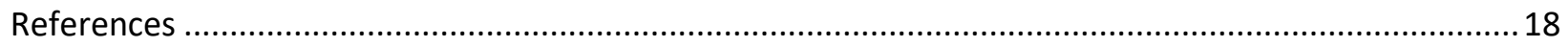




\title{
Multiple States of Nitrile Hydratase from Rhodococcus equi TG328-2: Structural and Mechanistic Insights from Electron Paramagnetic Resonance and Density Functional Theory Studies
}

\author{
Natalia Stein \\ Department of Physics, Marquette University, Milwaukee, WI \\ Natalie Gumataotao \\ Department of Chemistry, Marquette University, Milwaukee, WI \\ Department of Chemistry and Biochemistry, Loyola University, Chicago, IL \\ Natalia Hajnas \\ Department of Chemistry and Biochemistry, Loyola University, Chicago, IL \\ Rui Wu \\ Department of Chemistry and Biochemistry, Loyola University, Chicago, IL \\ K. P. Wasantha Lankathilaka \\ Department of Chemistry, Marquette University, Milwaukee, WI \\ Uwe T. Bornscheuer \\ Institute of Biochemistry, Department of Biotechnology \& Enzyme Catalysis, Greifswald \\ University, Germany \\ Dali Liu \\ Department of Chemistry and Biochemistry, Loyola University, Chicago, IL \\ Adam T. Fiedler \\ Department of Chemistry, Marquette University, Milwaukee, WI \\ Richard C. Holz \\ Department of Chemistry, Marquette University, Milwaukee, WI \\ Brian Bennett \\ Department of Physics, Marquette University, Milwaukee, WI
}




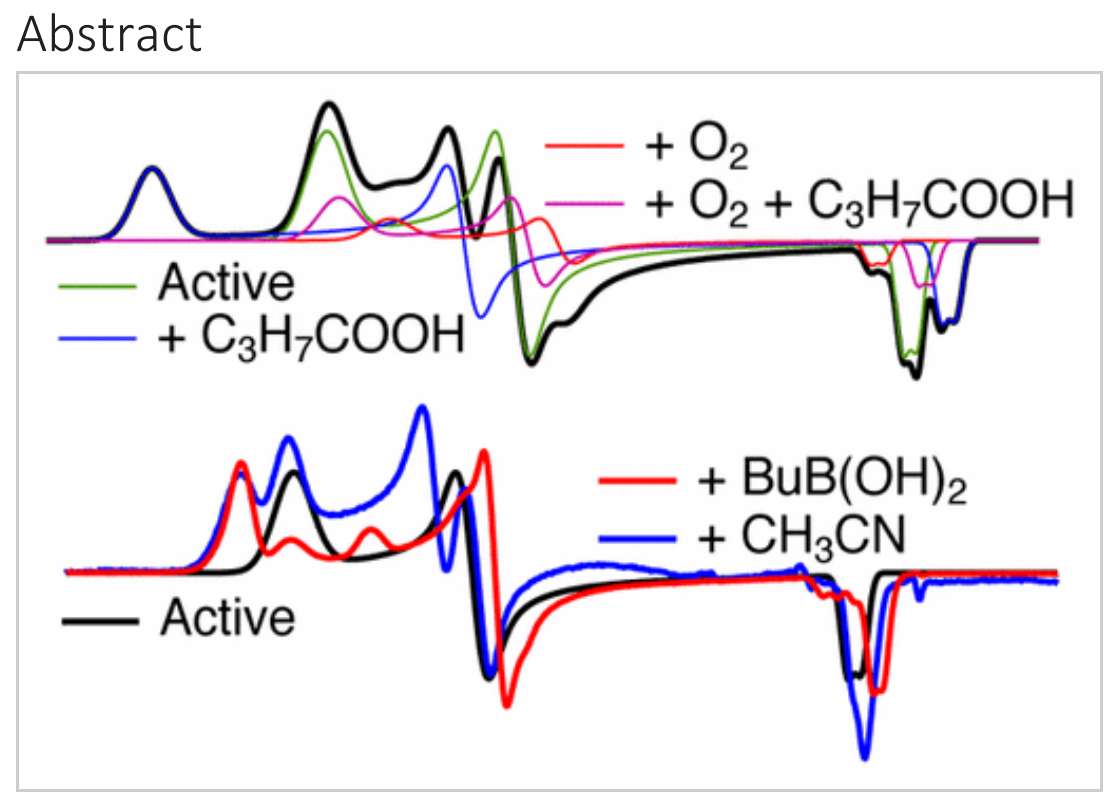

Iron-type nitrile hydratases (NHases) contain an Fe(III) ion coordinated in a characteristic "claw setting" by an axial cysteine thiolate, two equatorial peptide nitrogens, the sulfur atoms of equatorial cysteinesulfenic and cysteine-sulfinic acids, and an axial water/hydroxyl moiety. The cysteine-sulfenic acid is susceptible to oxidation, and the enzyme is traditionally prepared using butyric acid as an oxidative protectant. The as-prepared enzyme exhibits a complex electron paramagnetic resonance (EPR) spectrum due to multiple low-spin $(S=1 / 2) \mathrm{Fe}(\mathrm{III})$ species. Four distinct signals can be assigned to the resting active state, the active state bound to butyric acid, an oxidized Fe(III)-bis(sulfinic acid) form, and an oxidized complex with butyric acid. A combination of comparison with earlier work, development of methods to elicit individual signals, and design and application of a novel density functional theory method for reproducing $\mathbf{g}$ tensors to unprecedentedly high precision was used to assign the signals. These species account for the previously reported EPR spectra from Fe-NHases, including spectra observed upon addition of substrates. Completely new EPR signals were observed upon addition of inhibitory boronic acids, and the distinctive $g_{1}$ features of these signals were replicated in the steady state with the slow substrate acetonitrile. This latter signal constitutes the first EPR signal from a catalytic intermediate of NHase and is assigned to a key intermediate in the proposed catalytic cycle. Earlier, apparently contradictory, electron nuclear double resonance reports are reconsidered in the context of this work.

The high industrial demand for amides, particularly polyacrylamide and nicotinamide, is traditionally met through manufacturing processes that require high acidity, high pressure, and high temperature $\left(200-400{ }^{\circ} \mathrm{C}\right)$, along with metal catalysts such as Raney copper.(1) These industrial reactions are economically inefficient and do not yield the pure amide product, because of the formation of unwanted acid side products. Nitrile hydratases (NHases) catalyze the stereo- and regiospecific hydration of nitriles to their corresponding amides under ambient conditions and physiological $\mathrm{pH} .(1)$ Therefore, NHases have been utilized as industrial biocatalysts to produce, most notably, acrylamide and nicotinamide in single-step reactions, with the elimination of the formation of acid side products.(2) NHases have also proven to be useful in the bioremediation of chemical and wastewater runoff, specifically for the hydration of the pesticide bromoxynil.(3) Despite the relative success of the use of 
NHase in manufacturing and bioremediation, several details of their catalytic mechanism remain poorly understood.

NHases are metalloenzymes that contain either a low-spin $(S=1 / 2) \mathrm{Fe}(\mathrm{III})$ (Fe-type NHases) or a low-spin $(S=0)$ Co(III) (Co-type NHases) ion.(1) X-ray crystallographic studies of NHases revealed that they are $\alpha_{2} \beta_{2}$ heterotetramers with the active-site metal ion coordinated in a characteristic "claw setting" by an axial cysteine thiolate, two equatorial peptide nitrogens, equatorial sulfur atoms that are posttranslationally modified to cysteine-sulfenic $(\mathrm{CysSOH})$ and cysteine-sulfinic $\left(\mathrm{CysSO}_{2} \mathrm{H}\right)$ acids, and a labile axial water or hydroxyl moiety (Scheme 1 and Figure 1).(4, 5) This geometry is conserved among all Fetype and Co-type NHase enzymes. $(1,6-8)$ The protonation states of the axial Cys and the posttranslationally modified cysteine-sulfenic and cysteine-sulfinic acids were suggested to be Cys-S-, $\mathrm{CysSOH}$, and a $\mathrm{CySSO}_{2}-$ based on sulfur K-edge EXAFS and geometry-optimized density functional theory (DFT) calculations.(9) The post-translational oxidation of the two equatorial cysteine thiolate moieties to sulfenic and sulfinic acids is essential for catalytic activity.(10-12)

\section{Scheme 1. Proposed Structure for the BuBA Species of NHase}

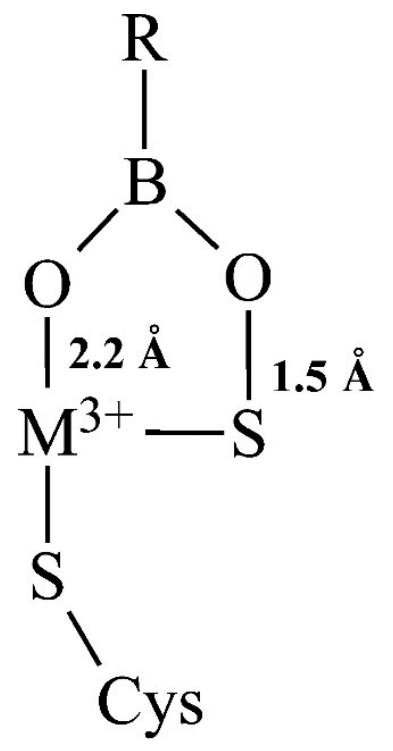



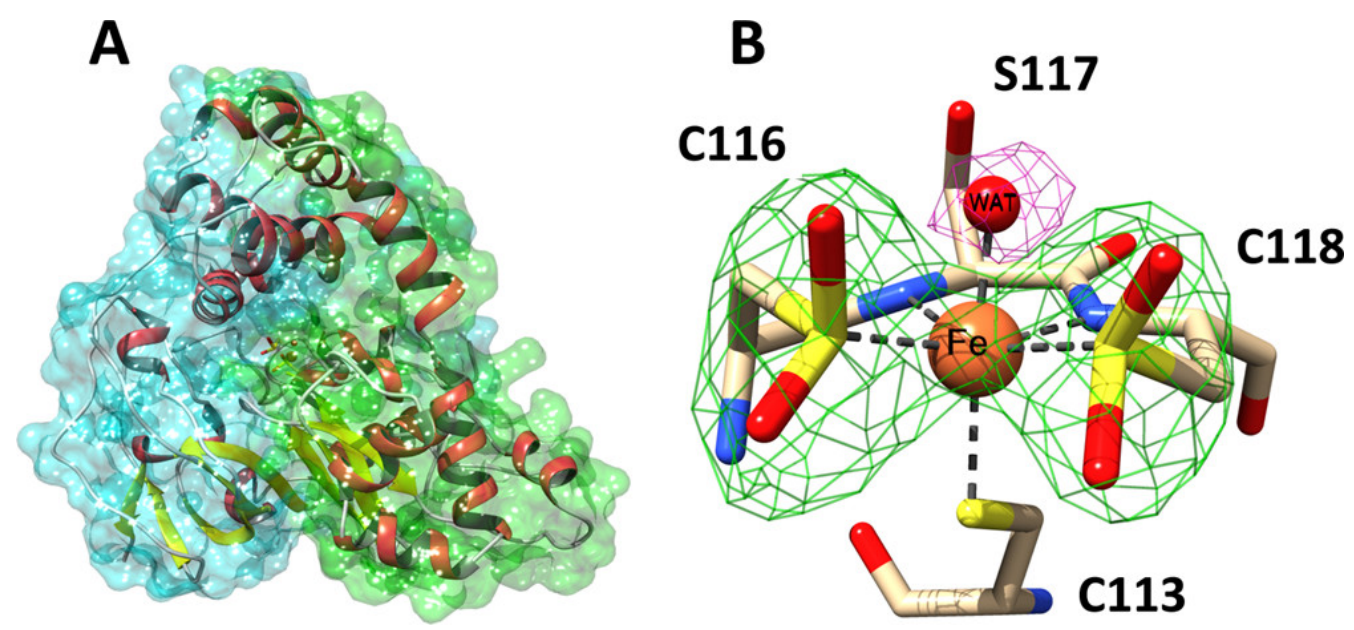

Figure 1. Structure of ReNHase-TG328-2. (A) Overall structure of ReNHase. The two subunits of the $\alpha \beta$ heterodimer are shown with superimposed green $(\alpha)$ and cyan $(\beta)$ surface plots. $(B)$ Iron center of the enzyme. The iron coordination geometry is represented by dashed lines. A difference omit map $\left(F_{0}-F_{c}\right)$ for the coordinated water is shown at the $4 \sigma$ level (magenta), and the omit map for the two cysteine-sulfinic acid sulfinate groups is shown at $5 \sigma$ (green).

Electron paramagnetic resonance (EPR) studies have provided a wealth of mechanistic information about metalloenzymes and have been reported for several Fe-type enzyme species, including the resting states of NHases from Rhodococcus (formerly Brevibacterium) R312 (RhNHase-R312) and Pseudomonas chloraphis 23 (PcNHase-23),(13) as well as from NHases following various treatments with substrates or inhibitors,(14) and Fe-type NHases after photoactivation of an as-prepared NO-inhibited form.(15) A variety of EPR signals have been observed, often consisting of multiple species with overlapping spectra. However, in cases in which signals could be deconvoluted, they all appear to be rhombic with the following $g$ values: $g_{1} \approx 2.2-2.3, g_{2} \approx 2.15$, and $g_{3} \approx 1.97-1.99$. In a description of the EPR spectrum of the NHase from Rhodococcus erythropolis N771 (RhNHase-N771), Odaka, Solomon, and co-workers ascribed two of the EPR signals commonly observed in Fe-type NHases to the active resting form ("NHaseAq") and to a butyric acid complex ("NHaseBA"). These assignments were substantiated by complementary magnetic circular dichroic and DFT information.(16) An additional set of less welldefined and $\mathrm{pH}$-dependent signals were assigned to "NHaseOx" forms of the enzyme, in which the equatorial cysteine-sulfenate had been oxidized to sulfinate. A survey of the available EPR data on NHase enzymes reveals that complexation of the enzyme by either carboxylic acids or certain amides appears to provide signals with $g_{1}$ values at the higher end of the observed range, whereas attempts to elicit signals with substrates or substrate analogues provided signals that were indistinguishable from those of the active enzyme.

In this study, we provide new insight into the active site and catalytic mechanism of the Fe-type NHase from Rhodococcus equi TG328-2 (ReNHase-TG328-2), using EPR, DFT, and X-ray crystallography. The complex EPR signals exhibited by the as-prepared ReNHase-TG328-2 enzyme have been completely assigned, and the nature of the oxidized and butyric acid complexes has been investigated. We have determined conditions for generating single species of ReNHase-TG328-2 and have characterized a hitherto unreported oxidized enzyme complex with butyric acid. This latter species exhibited a wellresolved splitting on the $g_{3}$ turning point, also evident in other signals upon careful inspection, that is assigned to a nonexchangeable proton. Electron nuclear double resonance (ENDOR) spectroscopy 
suggests that this splitting is due to a C- $\beta$ proton from the axial cysteine ligand. EPR data and DFT calculations prompted a thorough reexamination of previous assignments of the $\mathrm{Fe}^{3+}$ axial ligand in the active resting species. Finally, we characterized the EPR spectra of the enzyme in the steady state with a slow substrate and the inhibited complexes of ReNHase-TG328-2 with boronate inhibitors. Comparison with the observed EPR spectrum of ReNHase-TG328-2 in the steady state with acetonitrile suggests that one of the intermediates in hydration of acetonitrile is structurally similar to the boronate complexes and provides support for the direct binding of the substrate to the active-site Fe(III), the formation of a cyclic intermediate, and the role of the sulfenic acid ligand as the catalytic nucleophile.

\section{Materials and Methods}

\section{Preparation and Assay of ReNHase-TG328-2}

ReNHase-TG328-2 was encoded by a previously described plasmid(17) and isolated and assayed as previously described in detail.(18)

$X$-ray Crystallography is described in detail in the Supporting Information (T1). Data were processed using HKL2000.(19) Molecular replacement was performed against CtNHase [Protein Data Bank (PDB) entry 4FM4](20) using Phaser.(21) Model building and refinement were performed with Coot,(22) CCP4,(23) and Phenix.(24) Refinement statistics are listed in Table S1.

\section{Electron Paramagnetic Resonance}

X-Band EPR spectra were recorded at either $30 \mathrm{~K}$ and $0.1 \mathrm{~mW}$ on a Bruker EMXplus or an EleXsys E600 spectrometer, equipped with ER4116DM $(\sim 9.6 \mathrm{GHz})$ and ER4123-SHQE $(\sim 9.4 \mathrm{GHz})$ resonators, an Oxford Instruments ESR900 helium flow cryostat, and an Oxford Instruments ITC503 temperature controller, or $77 \mathrm{~K}$ and $1 \mathrm{~mW}$ on a Bruker EMX-AA spectrometer equipped with an ER4112-SHQ resonator ( $\sim 9.5 \mathrm{GHz})$. L-Band EPR was recorded at $1.85 \mathrm{GHz}$ and $120 \mathrm{~K}$ on a home-built spectrometer equipped with a 1-2 GHz octave-band bridge and a two-loop, one-gap resonator. $(25,26)$ Precise frequencies were recorded in each case (EIP 548A Microwave Frequency Counter). Spectra were characterized in terms of $g$ values, $\mathbf{g}$ anisotropy $\left(g_{1}-g_{3}\right)$, and $\mathbf{g}$ rhombicity, $R_{\mathrm{g}}$, where $R_{\mathrm{g}}=1-\left(\mid g_{1}+g_{3}-\right.$ $\left.2 g_{2} \mid\right) /\left(g_{1}-g_{3}\right)$, and therefore, $R_{\mathrm{g}}=0$ for the axial cases of $g_{1}=g_{2}$ and $g_{2}=g_{3}$ and $R_{\mathrm{g}}=1$ for the most rhombic case in which $g_{1}-g_{2}=g_{2}-g_{3}$. Fractional spin intensities were estimated by least-squares fitting

of simulated spectra (EasySpin(27)) to experimental data and absolute spin concentrations estimated by comparison to a standard sample containing $0.5 \mathrm{mM} \mathrm{Cu}(\mathrm{II}), 20 \mathrm{mM}$ imidazole, and $25 \mathrm{mM}$ HEPES (pH 7.5). Pulsed Davies ENDOR spectra were recorded at $10 \mathrm{~K}$ on a Bruker EleXsys E580 spectrometer equipped with an E560-P stochastic pulsed ENDOR accessory, an ENI A300 RF amplifier, and an EN4118X-MD-4W resonator.

\section{Computational Methods}

DFT calculations were performed using ORCA version 3.0 developed by Neese.(28) The Fe-type NHase calculations employed a truncated version of the active site derived from crystallographic data (PDB entry $2 \mathrm{AHJ})(29)$ and previous computational studies by Hopmann.(30) The computational models included the two conserved protonated arginine residues (Arg56 and Arg141) that form hydrogen bonds to the $\mathrm{CysSO}^{-}$and $\mathrm{CysSO}_{2}{ }^{-}$donors. The positions of carbon atoms attached to the protein backbone were fixed during geometry optimizations. The models possess an overall charge of zero and an $S=1 / 2$ spin state. All calculations utilized the PBE0 functional (i.e., the one-parameter hybrid version(31) of the 
Perdew-Burke-Erzerhoff functional(32)), which has proven to be accurate in prior studies of ironcontaining complexes.(33) Ahlrichs' valence triple- $(\mathrm{TZV})$ and TZV/J auxiliary basis sets were used in each case, with additional polarization functions on heavy atoms.(34-36) The computed Fe-ligand bond lengths for each model are summarized in Table S3, and atomic coordinates are provided (Tables S4-S8). TD-DFT calculations(37-39) provided absorption energies and intensities for 40 excited states within the Tamm-Dancoff approximation. $(40,41)$

Two different methods were employed to calculate $g$ values for the active-site models. The first approach involved solving the coupled-perturbed self-consistent field (CP-SCF) equations, as implemented in ORCA, $(42,43)$ to assess the contribution of spin-orbit coupling $(\mathrm{SOC})$ to the $\mathbf{g}$ tensor. The second approach relied upon a series of equations, originally developed by Taylor,(44) that relate $g$ values to ligand field parameters for low-spin Fe(III) systems. According to Taylor, the three $g$ values are related to the coefficients $\left(a, b\right.$, and $c$ ) of the $\mathrm{d}_{y z}, \mathrm{~d}_{x z}$, and $\mathrm{d}_{x y}$ orbitals, respectively, in the singly occupied orbital, as indicated in eqs 1-3:

$$
\begin{aligned}
& g_{z}=2\left[(a+b)^{2}-c^{2}\right](1) \\
& g_{y}=2\left[(a+c)^{2}-b^{2}\right](2) \\
& g_{x}=2\left[a^{2}-(b+c)^{2}\right](3)
\end{aligned}
$$

This mixing of the " $\mathrm{t}_{2 \mathrm{~g}}$ " orbitals is due to spin-orbit coupling. Therefore, the $g$ values can be used to calculate the tetragonal splitting $(\Delta)$ between the $d_{x y}$ orbital and the $d_{x z} / d_{y z}$ pair and the rhombic splitting $(V)$ between the $d_{x z}$ and $d_{y z}$ orbitals, according to eqs 4 and 5 .

$$
\begin{gathered}
\frac{V}{\Lambda_{F e}}=\frac{g_{x}}{g_{y}+g_{z}}+\frac{g_{y}}{g_{z}-g_{x}}(4) \\
\frac{\Delta}{\Lambda_{F e}}=\frac{g_{x}}{g_{z}+g_{y}}+\frac{g_{z}}{g_{y}-g_{x}}-\frac{V}{2 \Lambda_{F e}}(5)
\end{gathered}
$$

where $\lambda_{\mathrm{Fe}}$ is the SOC parameter. In this study, the $\Delta$ and $V$ values of each Fe-type NHase model were estimated from $d-d$ transition energies generated by the corresponding TD-DFT calculations. The three $g$ values in eqs 4 and 5 were then varied until the resulting $\Delta$ and $V$ energies matched those predicted by TD-DFT. The computed $g$ values were required to correspond to a normalized orbital, such that $a^{2}+b^{2}+$ $c^{2}=1$. By applying this approach to a series of test complexes, we determined that a $\lambda_{\mathrm{Fe}}$ value of $460 \mathrm{~cm}^{-}$ ${ }^{1}$ provides the best agreement between experimental and computed $g$ values. It is important to note that the three $g$ values in eqs $1-5$ can be either positive or negative. The correct signs of the $g$ values correspond to the molecular axis system in which the value of $|V / \Delta|$ is $<^{2} / 3$. (44) For the Fe-type NHase systems discussed here, this criterion is met when $g_{x}$ is negative, $g_{y}$ is positive, and $g_{z}$ is negative; however, only the absolute $g$ values are reported. 


\section{Results}

\section{X-ray Crystallography}

The active-site Fe(III) ion in ReNHase-TG328-2 is identical to that in other Fe-type NHases. Bond distances for Fe(III) ligands are similar to those previously reported for Fe-type NHases and are summarized in Table S2.

\section{EPR Spectroscopy}

\section{ReNHase-TG328-2 Prepared Aerobically in the Presence of Butyric Acid}

Fe-type NHase enzymes are typically prepared aerobically, necessitating the use of the competitive inhibitor butyric acid to prevent loss of activity. Butyric acid impedes oxidation of the active-site cysteine-sulfenic acid to cysteine-sulfinic acid. Procedures subsequently employed with the intention of removing butyric acid include column chromatography, dialysis, or, as here, three or more cycles of buffer exchange by ultrafiltration.

The enzyme thus prepared exhibited a complex EPR spectrum that differed from preparation to preparation in the relative intensity of each of the turning points at the reproducible resonant fields characteristic for ReNHase-TG328-2 (Figure 2). Three distinct rhombic species were identified by inspection and simulation and are termed $\mathrm{Na}, \mathrm{N} b$, and $\mathrm{Nc}$ [this notation is distinct from that $\left(\mathrm{NH}_{\mathrm{A}}, \mathrm{NH}_{\mathrm{C}}\right.$, etc.) employed elsewhere]. $\mathrm{N} a$ was the most anisotropic species observed and is characterized by $g_{1}, g_{2}$, and $g_{3}$ values of $2.281,2.150$, and 1.974 , respectively. A second well-defined species, $\mathrm{N} b$, exhibited $g_{1}$, $g_{2}$, and $g_{3}$ values of 2.206, 2.131, and 1.987, respectively. The $\mathbf{g}$ tensor for $\mathrm{N} b\left(g_{1}-g_{3}=0.219\right)$ was less anisotropic than that of $\mathrm{Na}\left(g_{1}-g_{3}=0.307\right)$ and exhibited higher axial symmetry (equivalent to lower rhombicity; $R_{\mathrm{g}}$ values for $\mathrm{N} a$ and $\mathrm{N} b$ were 0.85 and 0.69 , respectively). Conversion of the $\mathrm{Na}$ complement of the spectrum to the $\mathrm{N} b$ form was observed upon the addition of the substrate methacrylonitrile, but the conversion was not dependent on an active substrate and was also elicited by the addition of the innocent agent imidazole. Addition of $100 \mathrm{mM}$ imidazole to a sample of the butyric acid-complexed ReNHase-TG328-2 elicited a change from a spectrum due essentially to $\mathrm{N} a$ to one due almost completely to $\mathrm{Nb}$ (Figure 3 ). A third triad of turning points, evident as small peaks or shoulders labeled $c$ in Figure 2, are less definitive but consistent with a third rhombic $S=1 / 2$ species, Nc, with $g_{1}, g_{2}$, and $g_{3}$ values of $2.180,2.114$, and 1.998 , respectively, a $g_{1}-g_{3}$ value of 0.182 , and $R_{\mathrm{B}}$ of 0.73 . Nc was elicited deliberately at the expense of $\mathrm{Nb}$ by exposure of the enzyme to air (Figure $4 \mathrm{C}$ ). The $\mathrm{pH}$ dependence of the EPR spectrum of ReNHase-TG328-2 differed from that reported for $R h N H a s e-$ $\mathrm{N771}(16)$ and is described in detail in the text (T2) supporting Figure S1. Briefly, the pH dependence of the observed spectrum was appreciably weaker for ReNHase-TG328-2. Two distinct species were deconvoluted by difference, one at a higher $\mathrm{pH}$ corresponding to the $\mathrm{N} b$ signal and one at a lower $\mathrm{pH}$ that was most similar, though not identical, to the Nc form. 


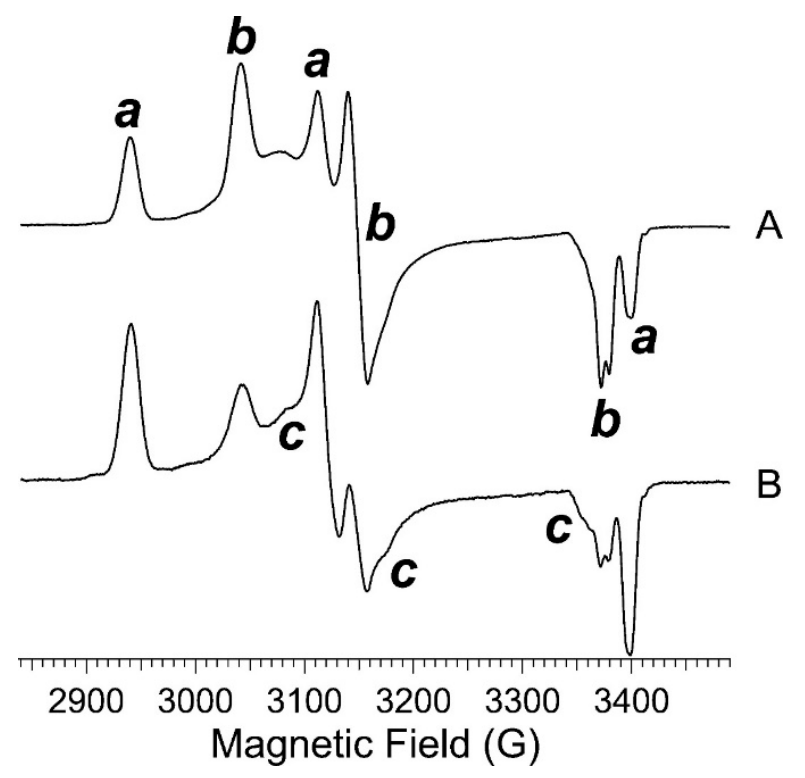

Figure 2. Representative EPR spectra of two preparations of ReNHase-TG328-2, in 50 mM HEPES buffer (pH 7.5) and residual butyric acid from the purification procedure, recorded at $9.4 \mathrm{GHz}, 30 \mathrm{~K}$, and a microwave power of 0.1 $\mathrm{mW}$. Labels $a-c$ denote turning points due to distinct species $\mathrm{Na}-\mathrm{Nc}$, respectively, which differ in proportion in spectra $A$ and $B$.

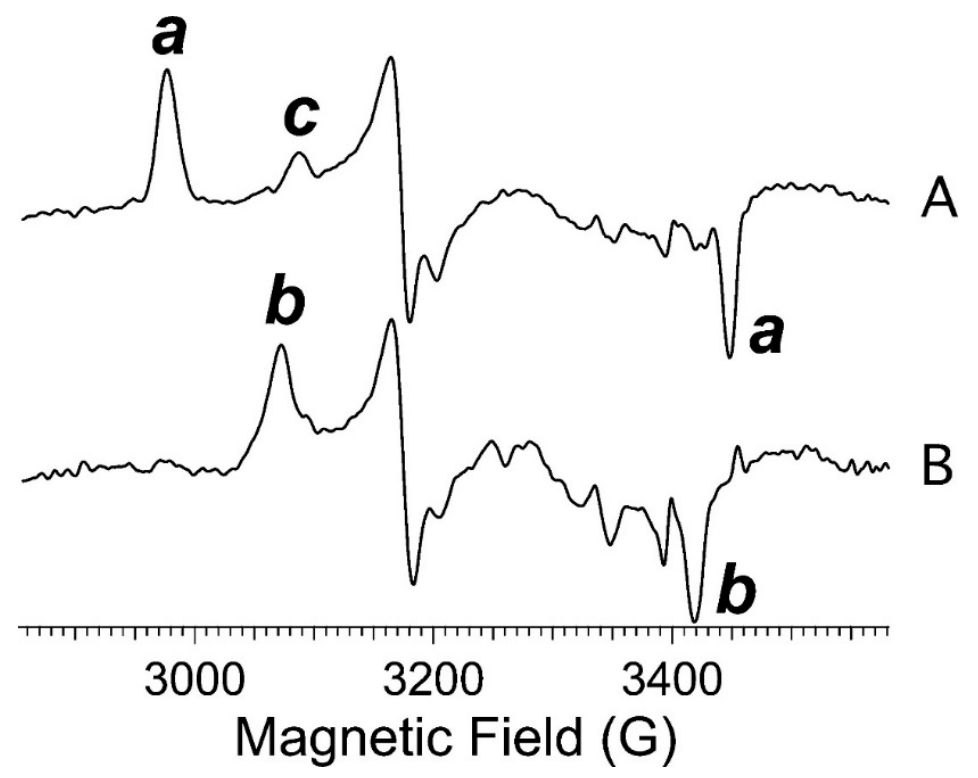

Figure 3. (A) EPR spectrum of ReNHase-TG328-2 in 50 mM HEPES buffer (pH 7.5) containing 5 mM butyric acid. (B) EPR spectrum of ReNHase-TG328-2 in 50 mM HEPES buffer (pH 7.5) containing 5 mM butyric acid and 100 mM imidazole. Spectra were recorded at $9.5 \mathrm{GHz}, 77 \mathrm{~K}$, and a microwave power of $1 \mathrm{~mW}$. Labels $a-c$ denote turning points due to distinct species $\mathrm{Na}-\mathrm{Nc}$, respectively. 


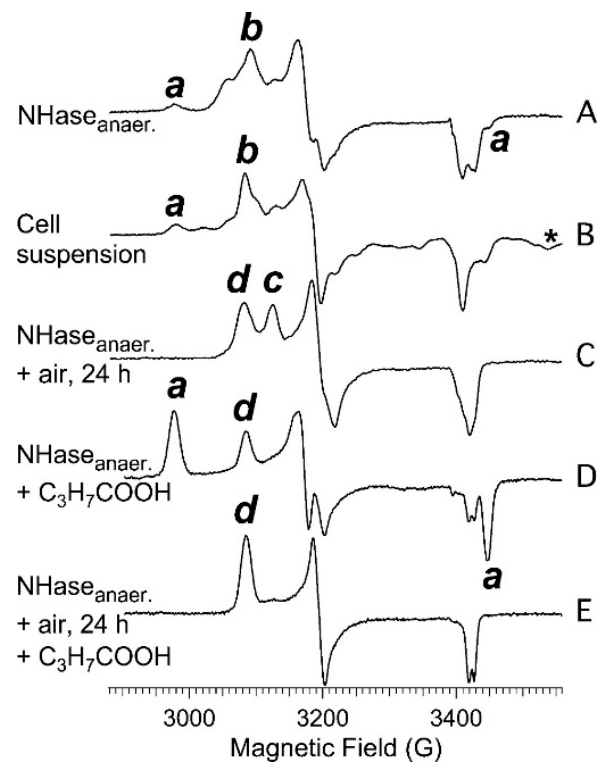

Figure 4. EPR spectra from (A) NHase following anaerobic anion-exchange chromatography using an elution buffer free of butyric acid, (B) a suspension of the cells from which NHase was isolated, which was not exposed to butyric acid, (C) an aliquot of sample A following exposure to air for $24 \mathrm{~h}$ at $25^{\circ} \mathrm{C}$, (D) an aliquot of sample $A$ to which 20 $\mathrm{mM}$ butyric acid was added, and (E) an aliquot of sample $B$ to which $20 \mathrm{mM}$ butyric acid was added. Labels $a-d$ denote turning points due to distinct species $\mathrm{Na}-\mathrm{Nd}$, respectively. The asterisk denotes the resolved $\left|m_{\mathrm{s}}, m_{1}\right\rangle=$ $| \pm 1 / 2,-3 / 2\rangle$ line of a signal due to $S=5 / 2, I=5 / 2{ }^{55} \mathrm{Mn}(\mathrm{II})$, assigned on the basis of the corresponding $\left| \pm^{1} / 2,-5 / 2\right\rangle$ line $90 \mathrm{G}$ upfield (not shown). Spectra were recorded at $9.5 \mathrm{GHz}, 77 \mathrm{~K}$, and a microwave power of $1 \mathrm{~mW}$.

\section{Anaerobic Preparation, Air Oxidation, and Butyric Acid Exposure of ReNHase-}

\section{TG328-2}

In an attempt to understand the chemical origins of the EPR-detected species and to develop protocols for isolating ReNHase-TG328-2 in each of its putative homogeneous states, ReNHase-TG328-2 was prepared by performing the chromatography steps in an anaerobic glovebox, and butyric acid was removed by a final anion-exchange step with an eluting buffer devoid of butyric acid. The resulting spectrum (trace A, Figure 4) was substantially due to the $\mathrm{Nb}$ form but contained traces of $\mathrm{Na}$, $\mathrm{Nc}$, and a novel signal termed $N d$, characterized by turning points labeled $d$ in Figure 4 with $g_{1}, g_{2}$, and $g_{3}$ values of $2.201,2.125$, and 1.982, respectively. The appearance of $\mathrm{N} a$ appears to be unrelated to the enzyme preparative procedure, as the EPR spectrum of a suspension of the cell culture from which ReNHaseTG328-2 was isolated, which had never been exposed to butyric acid, also exhibited the Na signal at approximately the same, albeit low, relative intensity (Figure 4B).

Upon incubation of an aliquot of the isolated NHase corresponding to Figure $4 \mathrm{~A}$ in air for $24 \mathrm{~h}$ at ambient temperature $\left(\approx 25^{\circ} \mathrm{C}\right)$, the $\mathrm{N} b$ component was extinguished and replaced by roughly equal amounts of $\mathrm{Nc}$ and the newly discovered $\mathrm{Nd}$ (Figure $4 \mathrm{C}$ ). The sample exhibited no measurable catalytic activity, and a longitudinal study of ReNHase-TG328-2 purified in an anaerobic glovebox showed that NHase activity decayed exponentially in air at $4{ }^{\circ} \mathrm{C}$ with a $\tau$ of $\approx 16 \mathrm{~h}$ (Figure 5). The addition of $20 \mathrm{mM}$ butyric acid to each of the samples that exhibited the spectra shown in traces $A$ and $C$ of Figure 4 elicited the spectra shown as traces $\mathrm{D}$ and $\mathrm{E}$, respectively. 


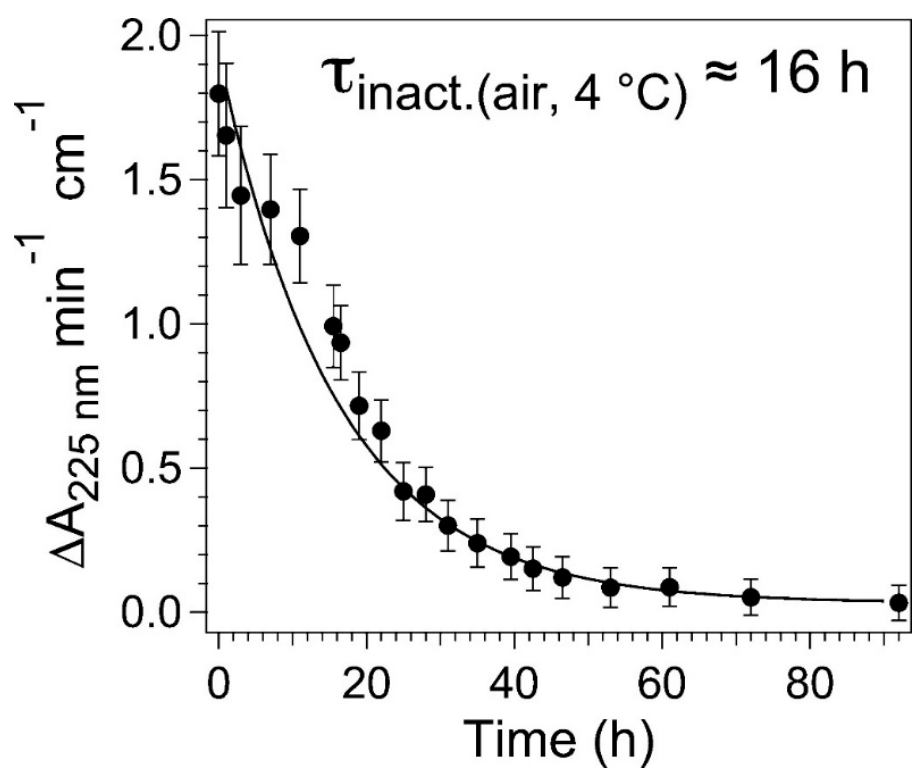

Figure 6. Pulsed Davies ENDOR spectra (top) recorded on the sample of Figure 4, Trace D, at $9.8 \mathrm{GHz}$ and $10 \mathrm{~K}$. Spectra shown were recorded at the $g_{1}, g_{2}$, and $g_{3}$ resonant fields, and $\pm A / 2$ nonexchangeable ${ }^{1} \mathrm{H}$ resonance pairs are labeled $a-c$, respectively. EPR spectrum (bottom) of the sample of Figure 4D, recorded with a nonsaturating microwave $\mathbf{B}_{1}$ field, at $1.85 \mathrm{GHz}$ and $120 \mathrm{~K}$.

\section{ReNHase-TG328-2 upon Incubation with Alkylboronate Inhibitors}

Information about the binding of BuBA and PBA on the Fe-type ReNHase-TG328-2 enzyme was sought via EPR, and representative spectra are shown in Figure 7. For reference, the as-prepared spectrum containing the $\mathrm{N} a$ signal from the butyric acid complex, the active $\mathrm{N} b$ species, and some oxidized $\mathrm{Nc}$ is reproduced from Figure 2 as Figure 7A (note that the magnetic field scale has been adjusted to account for different microwave frequencies, as mentioned in the figure legend). The EPR spectrum of ReNHaseTG328-2 following turnover with methacrylonitrile followed by buffer exchange exhibited the active $\mathrm{N} b$ signal but no signal due to the $\mathrm{Na}$ species (Figure 7B). Upon subsequent incubation of the sample shown in Figure 7B with either BuBA (Figure 7D) or PBA (Figure 7E), the EPR spectra become markedly more rhombic, with the dominant species exhibiting $g_{1}, g_{2}$, and $g_{3}$ values of 2.235, 2.128, and 1.985, respectively. A residual component, with $g_{1}$ indistinguishable from that of the active unreacted $\mathrm{Nb}$ species, was additionally observed in both spectra. A third, otherwise uncharacterized, resonance at $g=$ 2.180 was observed in the EPR spectrum of the sample containing BuBA, but not in the spectrum of the sample containing PBA. The dominant species is significantly more rhombic than that observed when, e.g., imidazole was added with conversion of $\mathrm{Na}$ to $\mathrm{Nb}$. Perhaps it is mechanistically significant that the $g_{1}$ value of the dominant species observed in the boronic acid complexes was reproduced in the spectrum obtained upon incubation of active NHase with the very slow substrate acetonitrile (Figure 7C). 


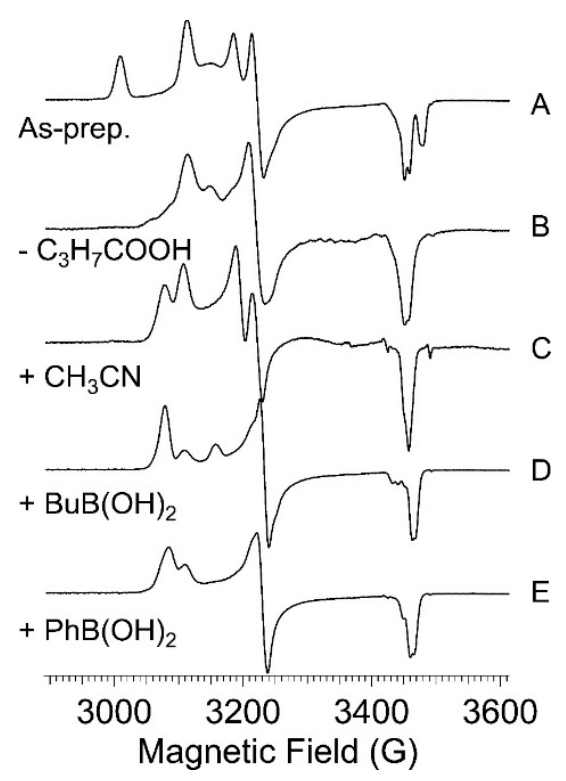

Figure 7. EPR spectra of (A) as-prepared NHase, (B) NHase with butyric acid removed, (C) sample B stirred with 50 $\mathrm{mM}$ acetonitrile for $10 \mathrm{~s}$ and frozen in isopentane at $-100{ }^{\circ} \mathrm{C}$, (D) NHase incubated with $10 \mathrm{mM}$ 1-butaneboronic acid at $25^{\circ} \mathrm{C}$ for $10 \mathrm{~min}$, and (E) NHase incubated with $10 \mathrm{mM}$ phenylboronic acid at $25^{\circ} \mathrm{C}$ for $10 \mathrm{~min}$.

\section{Density Functional Theory Calculations}

The relationship between the active-site structure and observed EPR features was further examined with computational methods. Five truncated models of the Fe-type NHase active site were generated, corresponding to the putative intermediates observed by EPR: NHase ${ }^{\mathrm{Aq}}$ (a model corresponding to $\mathrm{N} b$ ),

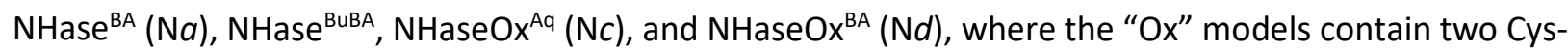
$\mathrm{SO}_{2}{ }^{-}$ligands. The "BA" and "Aq" structures feature metal-bound acetic acid (instead of butyrate) and $\mathrm{H}_{2} \mathrm{O}$, respectively, trans to the thiolate donor, and the "BuBA" structure [I (Scheme 1)] was based on the structure (PDB entry 4OB2) obtained from X-ray diffraction of crystals of Co-type PtNHase treated with BuBA.(45) The $g$ values were initially calculated using the coupled-perturbed self-consistent field (CPSCF) equations for spin-orbit coupling (SOC), $(42,43)$ as implemented in the ORCA computational package. However, as shown in Table 1 , the resulting $g$ values do not adequately reproduce the experimental values, a finding consistent with prior attempts to apply this methodology to low-spin Fe(III) systems. $(16,46)$ In particular, DFT consistently underestimates the $g_{1}$ values and provides $g_{3}$ values of $>2.0$, whereas the experimental $g_{3}$ value is always $<2.0$. We therefore developed an alternate approach that utilizes equations originally derived by Taylor for low-spin ferric heme complexes.(44) For such " $\mathrm{t}_{2 \mathrm{~g}}{ }^{5}$ " systems, the $\mathrm{g}$ anisotropy principally arises from in-state SOC. Therefore, the three $g$ values are related to the SOC constant, $\lambda_{\mathrm{Fe}}$, and the energy splittings, $\Delta$ and $V$, between the $t_{2 \mathrm{~g}}$ orbitals (see Materials and Methods for more details). Generally, Taylor's equations are used to calculate $\Delta$ and $V$ from the experimental $g$ values. We surmised, however, that they could also be employed in the reverse fashion, to predict $g$ values based on ligand field transition energies (i.e., $\Delta$ and $V$ splittings) computed using time-dependent DFT (TD-DFT), and this approach was developed here. 
Table 1. Comparison of Experimental and Computed $g$ Values for Fe(III)-NHase Species

\begin{tabular}{|l|l|l|l|l|l|l|}
\hline EPR signal (NHase species) & method $^{\text {a }}$ & $\boldsymbol{g}_{\mathbf{1}}$ & $\boldsymbol{g}_{\mathbf{2}}$ & $\boldsymbol{g}_{\mathbf{3}}$ & $\boldsymbol{g}_{\mathbf{1}}-\boldsymbol{g}_{\mathbf{3}}$ & rmsd $^{\text {b }}$ \\
\hline $\mathrm{N} b\left(\mathrm{NHase}^{\mathrm{Aq}}\right)$ & EPR exp. & 2.206 & 2.131 & 1.987 & 0.219 & \\
\hline & CP-SCF & 2.169 & 2.103 & 2.007 & 0.162 & 0.029 \\
\hline & Taylor/DFT & 2.215 & 2.117 & 1.979 & 0.236 & 0.011 \\
\hline $\mathrm{Na}\left(\mathrm{NHase}^{\mathrm{BA}}\right)$ & EPR exp. & 2.281 & 2.150 & 1.974 & 0.307 & \\
\hline & CP-SCF & 2.193 & 2.120 & 2.007 & 0.186 & 0.057 \\
\hline & Taylor/DFT & 2.248 & 2.121 & 1.970 & 0.278 & 0.025 \\
\hline $\mathrm{Nc}\left(\mathrm{NHaseO}^{\mathrm{Aq}}\right)$ & EPR exp. & 2.180 & 2.114 & 1.998 & 0.182 & \\
\hline & CP-SCF & 2.175 & 2.097 & 2.014 & 0.161 & 0.014 \\
\hline & Taylor/DFT & 2.219 & 2.111 & 1.976 & 0.243 & 0.026 \\
\hline $\mathrm{Nd}\left(\mathrm{NHaseO}^{\mathrm{BA}}\right)$ & EPR exp. & 2.201 & 2.125 & 1.982 & 0.219 & \\
\hline & CP-SCF & 2.193 & 2.109 & 2.035 & 0.158 & 0.032 \\
\hline & Taylor/DFT & 2.230 & 2.121 & 1.974 & 0.256 & 0.018 \\
\hline $\mathrm{NHase}^{\text {BuBA }}$ & EPR exp. & 2.235 & 2.128 & 1.985 & 0.250 & \\
\hline & CP-SCF & 2.150 & 2.096 & 1.999 & 0.151 & 0.053 \\
\hline & Taylor/DFT & 2.202 & 2.106 & 1.980 & 0.222 & 0.023 \\
\hline
\end{tabular}

aEPR exp. indicates experimental values from EPR; see the main text for details regarding the CP-SCF and Taylor/DFT methods.

bRoot-mean-square deviation between computed and experimental $g$ values.

To evaluate its feasibility, this procedure was first applied to a series of Fe-type NHase model complexes generated by Kovacs, for which X-ray structures and EPR spectra were available.(51) As shown in Table S9, the $g$ values provided by the combined Taylor/TD-DFT approach (subsequently termed the "Taylor/DFT method") exhibit much better agreement with the experimental data than the values from the corresponding ORCA calculations do. The Taylor/DFT method yields $g_{3}$ values of $<2.0$ and replicates the changes in the $\mathbf{g}$ tensor that occur upon protonation and/or oxidation of the thiolate ligand. Significantly, these preliminary studies were used to optimize the $\lambda_{\mathrm{Fe}}$ value, which cannot be calculated directly, and a value of $460 \mathrm{~cm}^{-1}$ provided the best agreement between computed and experimental $g$ values. This $\lambda_{\mathrm{Fe}}$ value is very close to the free ion value. Although metal-ligand covalency is expected to lower $\lambda_{\mathrm{Fe}}$ to around $400 \mathrm{~cm}^{-1}$ for a low-spin ferric species, in our case, the higher $\lambda_{\mathrm{Fe}}$ value compensates for the fact that TD-DFT calculations systematically overestimate the energies of $d-d$ transitions by 20 $30 \%$.

Application of the Taylor/DFT method to the four active-site models of Fe-type NHase yielded $g$ values that are consistent with the experimental data, which is evident in the overall root-mean-square deviation (rmsd) of only 0.021 (Table 1). Significantly, the Taylor/DFT calculations well reproduce the anisotropy $\left(g_{1}-g_{3}\right)$ of the experimental $\mathbf{g}$ tensors, providing computed $g_{1}$ values of $>2.20$ and $g_{3}$ values of $<2.00$. This represents a significant improvement over values obtained via CP-SCF calculations, which exhibit a larger rmsd of 0.040 . However, it is important to note that both methods correctly predict the increase in $g_{3}$ upon substitution of $\mathrm{H}_{2} \mathrm{O}$ with $\mathrm{BA}$ (for the native and oxidized enzymes) as well as the decrease in $g_{3}$ upon conversion of $\mathrm{NHase}^{\mathrm{BA}}$ to $\mathrm{NHaseO}^{\mathrm{BA}}$. 


\section{Discussion}

\section{Origins of Multiple Chemical Species in As-Prepared ReNHase-TG328-2}

On the basis of (i) comparison with previously reported EPR signals from related NHases, (ii) the nature of treatments used to elicit individual signals and associated activity measurements, and (iii) Taylor/DFT calculations using structural models, the four signals from NHases prepared under standard conditions were assigned to distinct chemical species. The $g$ values for the $\mathrm{N} a$ signal are characteristic of butyric (or, more generally, carboxylic) acid complexes of Fe-type $\operatorname{NHases}(13,15,45)$ and indicate incomplete removal of bound carboxylic acid from the enzyme active site following its use as an antioxidation protectant. The signal was modeled well by Taylor/DFT, which reproduced the unusually anisotropic $\mathbf{g}$ tensor with unprecedented precision, using a model that included monodentate binding of carboxylic (acetic) acid to the Fe(III) ion in place of the water/hydroxyl that was crystallographically identified in the active enzyme. This model was suggested by the X-ray diffraction structure of a Co-type NHase complexed by butyric acid in which the ligand is bound through a carboxylic oxygen atom to the $\mathrm{Co}$ (III) ion. Further pieces of evidence for the assignment of $\mathrm{Na}$ are (i) the observation that NHase solutions containing butyric acid that exhibit the $\mathrm{Na}$ signal are inactive, (ii) the observation that the $\mathrm{Na}$ signal is converted to $\mathrm{N} b$ by scrupulous and anaerobic removal of butyric acid, and (iii) the observation that in NHase that exhibits $\mathrm{Na}$ after removal of exogenous, but not bound, butyric acid, the enzyme is active and exhibits the $\mathrm{N} b$ signal following turnover. This latter observation strongly suggests displacement of the competitive inhibitor butyric acid by substrate, leaving the active enzyme following turnover.

Therefore, the assignment of $\mathrm{Na}$ to a carboxylic acid complex of NHase is supported by widespread EPR data, including (i) its elucidation by addition of butyric acid, (ii) its elimination by displacement, and (iii) its diminution or nonappearance in preparations that either avoided or scrupulously removed butyric acid. The fact that the butyric acid form has no catalytic activity has been demonstrated, and the contention that the butyric acid complex includes replacement of the apical water/hydroxyl, and monodentate binding to the metal ion, is supported by (i) X-ray crystallographic data and (ii) the observation that the $\mathrm{Na}$ form can be converted to the active $\mathrm{Nb}$ form by cryogenic photolysis and subsequently re-formed by annealing. Additional support comes from the albeit circumstantial DFT evidence, particularly reproduction of the very high $g_{1}$ value. The one reported phenomenon that appears to be at odds with the assignment of $\mathrm{Na}$ is the earlier assignment of ENDOR-identified exchangeable protons in this species to the water/hydroxyl that occupies the sixth coordination site in the active form of the enzyme. $(5,47)$ A coordinated water/hydroxyl would not be expected in Na. Given the large amount of evidence from multiple investigators in favor of the assignment of $\mathrm{Na}$ to a carboxylic (butyric) acid complex and clear evidence from crystallography that a carboxylic oxygen binds the active metal ion in place of the water/hydroxyl, the simplest explanation for the ENDOR data is that the hitherto observed resonances are not, in fact, due to a coordinated water/hydroxyl but to nearby exchangeable protons. However, other possibilities exist,(48-50) and these are explored at length in section T3 of the Supporting Information. One final point of interest is the observation that the Na signal can be observed in the EPR spectrum of cells expressing NHase that had never been exposed to butyric acid (Figure 4B). This signal is initially very weak, but its intensity increases, proportionally, with time, indicating binding of endogenous carboxylic acids by NHase, at least in the Escherichia coli overexpression system. 
The species that exhibits the $\mathrm{N} b$ signal is assigned to the active form of the enzyme. This form of the enzyme exhibits maximal catalytic activity, is reversibly inhibited by butyric acid, and is irreversibly deactivated by exposure to oxygen. A species with $g$ values very similar to those of $\mathrm{N} b$ was assigned to the active "NHaseAq" species of RhNHase-N771(16) and was also observed in PcNHase-23.(13)

Taylor/DFT calculations based on the active enzyme model again reproduced the $\mathbf{g}$ tensor to unprecedented precision and were clearly very distinct from those of other structural models (Table 1). Conversion of $\mathrm{Na}$ to $\mathrm{N} b$ was demonstrated here by displacement of butyric acid with either substrate or the innocent ligand imidazole, and the enzyme could be isolated substantially as the active Nb form by scrupulous avoidance of butyric acid. Similar behavior was observed with the related $R h N H a s e-R 312$ enzyme; an Na-type signal with $g_{1}, g_{2}$, and $g_{3}$ values of $2.207,2.124$, and 1.984 , respectively, a $g_{1}-g_{3}$ values of 0.223 , and an $R_{\mathrm{B}}$ value of 0.74 was elicited upon addition of the substrate 2-

methylpropanenitrile (isobutyronitrile) to a preparation that hitherto expressed only the $\mathrm{Na}$ species associated with carboxylic acid; as is customary, the enzyme was prepared using butyric acid as a stabilizer.(13) For both the N771 and R312 enzymes, product release is the rate-limiting step in the reaction with substrates thus far investigated, and occupation of the proteinaceous binding site by the amide product likely prevents rebinding of residual butyric acid, as seen with imidazole.(18) An Nb-like signal from RhNHase-R312 was also observed upon cryogenic $(20 \mathrm{~K})$ illumination of the $\mathrm{N} a$ form, but the signal reverted to $\mathrm{Na}$ upon storage for 3 days at $77 \mathrm{~K}$.(14) This phenomenon suggests rebinding of butyric acid to $\mathrm{Fe}(\mathrm{III})$ following photolytic cleavage, presumably of an $\mathrm{Fe}-\mathrm{O}$ bond, and provides further, if circumstantial, evidence of the assignments of $\mathrm{N} a$ and $\mathrm{N} b$.

The species of NHase that exhibits the Nc signal is (i) present in aerobically isolated NHase preparations but not in anaerobically prepared enzyme, (ii) irreversibly inactive, and (iii) elicited by exposure to oxygen (air) in the absence of butyric acid. Its appearance is inhibited by the presence of butyric acid, and preparations of numerous NHase variants prepared with butyric acid exhibit only the $\mathrm{Na}$ signal and neither the active $\mathrm{N} b$ signal nor the oxidized $\mathrm{N} c$ signal. NHase that has been crystallized under aerobic conditions typically exhibits oxidation of the cysteine-sulfenic acid to cysteine-sulfinic acid.(45) Nc has an unusually low value for $g_{1},<2.2$, and the only other described examples of Fe-type NHase signals with $g_{1}$ values of $<2.2$ are those assigned to a species ("NHaseOx") of RhNHase-N771, in which the cysteinesulfenic acid was presumed to be oxidized to cysteine-sulfinic acid.(16) The traditional CP-SCF, uniquely for NHase species, provided a better overall fit for Nc than the Taylor/DFT method did. The Taylor/DFT parameters for $\mathrm{N} c$ were not markedly dissimilar from those for active $\mathrm{N} b$, suggesting that the electronic structure of the Fe(III) ion is not significantly perturbed by the replacement of cysteine-sulfenic by cysteine-sulfinic acid per se but that this replacement may result in other subtle changes in the activesite structure that are not reflected in the refinement of the model used for DFT or, given the otherwise superimposition of active and oxidized active-site X-ray structures, are within crystallographic resolution but nevertheless do impact the experimental $g$ values. Regardless, the chemical, crystallographic, and activity data for the assignment of this species are compelling.

The final signal observed in as-prepared NHase, $\mathrm{Nd}$, was only completely characterized as a result of its deliberate generation by treatment of the fully oxidized $(\mathrm{Nc})$ species with butyric acid. This unexpected reaction resulted in the transition from the spectra of Figure $4 \mathrm{C}-\mathrm{E}$. The $g$ values of $\mathrm{N} d$ were themselves unremarkable but were reproduced well by the Taylor/DFT method, assuming a structure analogous to that of $\mathrm{Na}$ but with the replacement of cysteine-sulfenic by cysteine-sulfinic acid. As for $\mathrm{Nc}$, the effect of the oxidation on the calculated $g$ values was not as great as on the experimental ones, but the direction 
of the shift of $g_{1}$ from the very large value for $\mathrm{N} a$ to a more modest value for $\mathrm{N} d$ was consistent with the experimental observation.

ENDOR data provided information about the origin of the EPR-detected hyperfine coupling. Lowfrequency EPR confirmed that the splitting was due to a single $I=1 / 2$ nucleus. An ENDOR coupling consistent with that was observed at $g_{3}$ and decreased slightly as the field was lowered but was essentially isotropic, indicating that a through-bond interaction between the proton and the Fe(III) ion was the dominant coupling mechanism. Taken together, these data suggest that the nonexchangeable proton responsible for the observed superhyperfine splitting of the $g_{3}$ EPR line is a C- $\beta$ proton from the axial cysteine ligand. The anisotropy of the coupling to an additional nonexchangeable proton, $b$, with $A$ values of 10,12 , and $7 \mathrm{MHz}$ at $g_{1}, g_{2}$, and $g_{3}$, respectively, explains the lack of superhyperfine resolution at $g_{1}$ and $g_{2}$ in the EPR spectrum and is likely due to the other cysteine C- $\beta$ proton. The Davies ENDOR approach employed here revealed couplings to these nonexchangeable protons that are significantly larger that any ${ }^{1} \mathrm{H}$ couplings reported in the previous ENDOR studies of NHase. $(5,47)$ (These are discussed in detail in paragraph T3 of the Supporting Information.)

In summary, EPR spectra of ReNHase-TG328-2 were found to exhibit four signals in the as-prepared state: the active species (Nb); an oxidized and permanently inactive Fe(III)-bis(sulfinic acid) form (Nc); a reversibly inactivated carboxylic acid-complexed form $(\mathrm{Na})$, arising from the use of butyric acid as a stabilizer during isolation and/or intracellular interaction of Fe-type NHase with endogenous carboxylic acids; and a permanently inactive form $(\mathrm{Nd})$ due to complexation of $\mathrm{Nc}$ with butyric acid. Careful analysis of the reported changes in EPR spectra of as-prepared Fe-type NHase enzymes upon the addition of substrates indicates that they do not involve any new species, such as putative catalytic intermediates, but are simply due to displacement of a carboxylic acid in the substrate binding pocket of the $\mathrm{Na}$ form by the reaction product. The signal that replaces the $\mathrm{Na}$ signal in the reaction steady state is indistinguishable from the $\mathrm{Nb}$ (resting active) form and is therefore due to the regenerated active site.

DFT calculations provided a deeper understanding of how changes in active-site structure cause shifts in $g$ values. The paramagnetic electron of the active-site $S=1 / 2 \mathrm{Fe}(\mathrm{III})$ ion in Fe-type NHase enzymes is located in an Fe $3 \mathrm{~d}_{x y}$-based molecular orbital (MO), where the coordinate system is determined by the orientation of the $\mathbf{g}$ tensor (Scheme 2). This singly occupied MO (SOMO) is the highest-energy $\mathrm{t}_{2 \mathrm{~g}}$ orbital due to strong $\pi$-antibonding interactions with both the thiolate (S1 in Scheme 1 ) and amidate (N1) donors. In contrast, the Fe $3 \mathrm{~d}_{x z}$ orbital experiences only one destabilizing $\pi$-interaction (with the N2 amidate), while the lowest-energy Fe $3 d_{y z}$ orbital is not involved in $\pi$-bonding. Thus, the rhombic nature of the $\mathbf{g}$ tensor of the low-spin Fe(III) ion in Fe-type NHase enzymes arises from the facial orientation of the thiolate and amidate donors. On the basis of our calculations, the increase in $\mathbf{g}$ anisotropy upon displacement of $\mathrm{H}_{2} \mathrm{O}$ by a carboxylic acid (e.g., acetic acid in the $\mathrm{BA}$ structural model) is largely due to an increase in the Fe1-N1 bond length of $\sim 0.04 \AA$, which lowers the energy gap between the $3 d_{x y}$-based SOMO and the $d_{x z} / d_{y z}$ pair. The coordinated acid forms a strong $\mathrm{H}$-bond with the CysSO- donor, and this orientation causes a steric clash between the alkyl group of the carboxylic acid and the Ser113 side chain, thereby pushing the N1 donor away from the Fe center (Table S3). A similar structural change occurs when carboxylic acid binds to the oxidized bis(sulfinic) active site. These calculations also suggest that the decrease in $\mathbf{g}$ anisotropy observed experimentally upon oxidation of the sulfenate ligand is due to the attendant shortening of the axial Fe-S1 bond (Table S3), which destabilizes the SOMO relative to the $d_{x z} / d_{y z}$ pair. 


\section{Scheme 2. Base Active-Site Model for DFT Calculations}

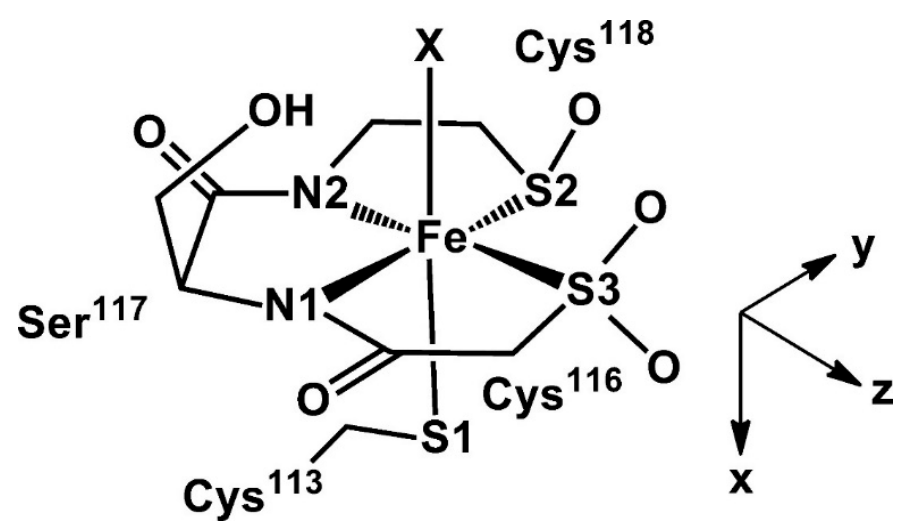

\section{EPR of ReNHase-TG328-2 upon Incubation with Alkylboronate Inhibitors}

Significant changes in the EPR spectrum were also observed upon the addition of boronic acid inhibitors to ReNHase-TG328-2. In these cases, however, new species that were clearly distinct from the $\mathrm{Na}-\mathrm{N} d$ quartet of signals that describe Fe(III) NHase spectra observed to date were identified. The boronic acid signals each exhibited a $g_{1}$ value intermediate between those of the $\mathrm{N} a$ and $\mathrm{N} b$ signals, and this feature was replicated by rapidly freezing a solution of ReNHase-TG328-2 with the slow substrate acetonitrile.

Insight into the catalytic mechanism of NHase enzymes has been obtained from the X-ray crystallographic study of the inhibited complexes of the Co-type NHase from Pseudonocardia thermophila (PtNHase) with 1-butaneboronic acid (BuBA) and phenylboronic acid (PBA).(45) Two structural models were obtained from X-ray diffraction data of PtNHase crystals soaked with BuBA and cocrystallized with BuBA. In the first (PDB entry 4OB2), the sulfenic acid oxygen atom was found covalently bound to the boron, indicating nucleophilic attack of the sulfenate oxygen atom on the empty $\mathrm{p}_{z}$ orbital of boron. An analogous model was obtained with PBA (PDB entry 4OBO), and these were interpreted as mimicking an intermediate in nucleophilic attack on the substrate. [Scheme 1 shows a cartoon of this BuBA species (I), and the corresponding proposed catalytic intermediate (II) is shown in Scheme 3.] A boronic acid oxygen atom was bound to the Co(III) ion, replacing water/hydroxyl, and the sulfenic acid sulfur atom remained bound to Co(III). The second model (PDB entry 4OB1) showed a weaker $\mathrm{S}-\mathrm{O}$ interaction, suggestive of BuBA dissociation mimicking product release. The dominant species is significantly more rhombic than that (i.e., $\mathrm{N} b$ ) observed following turnover of $\mathrm{Na}$-expressing NHase, or when imidazole is added to expel butyric acid, and strongly suggests binding of BuBA and PBA to the Fe(III) ion in the Fe-type NHase. It is tempting to speculate that the additional resonance that is present in the BuBA spectrum, but not in the PBA spectrum, is indicative of the second, more weakly interacting binding mode for BuBA indicated in the structural study. Most interestingly, the $g_{1}$ value of the dominant species observed in the boronic acid complexes was reproduced in the spectrum obtained upon incubating active NHase with the very slow substrate acetonitrile (Figure 7C). This may represent the first experimental evidence that the boronic acid complexes do indeed mimic steps in the reaction. On the basis of these data, then, the acetonitrile EPR signal is assigned to the cyclic intermediate (species II of Scheme 3) formed upon nucleophilic attack of the $\alpha \mathrm{Cys}-\mathrm{OH}$ sulfenic acid ligand on the $\mathrm{Fe}$ (III)-bound nitrile carbon atom of the substrate.(4) We tentatively propose that the minor component of the BuBA EPR signal, which is not observed with PBA, corresponds to the second crystallographically 
characterized conformation with BuBA (PDB entry 4OB1), in which the boronic O-Cys118 S interaction is much weakened, and was assigned to a mimic of the post-transition-state product release stage, corresponding to the species subsequent to species II in the " $A$ " and " $B$ " arms of Scheme 3.

\section{Scheme 3. Proposed Catalytic Cycle for NHase}

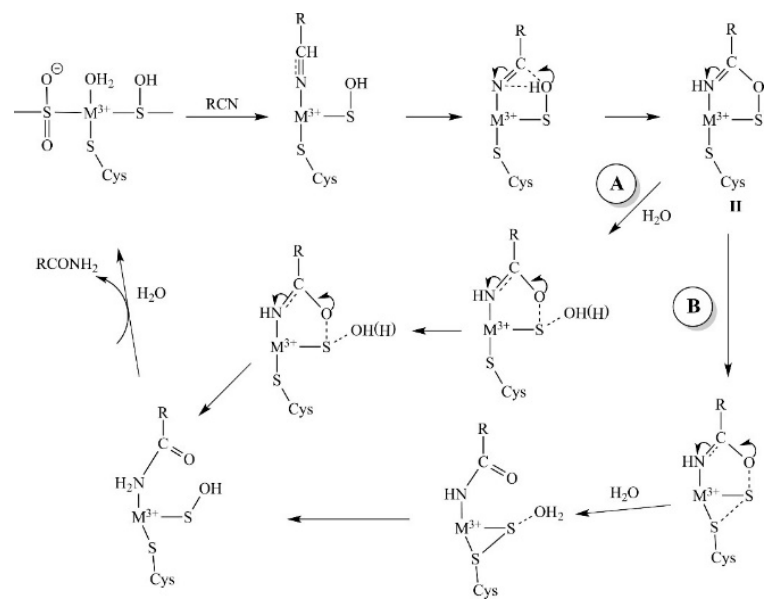

\section{Supporting Information}

The Supporting Information is available free of charge on the ACS Publications website at DOI: 10.1021/acs.biochem.6b00876.

- Details of X-ray crystallography, metric parameters for geometry-optimized models of the FeNHase active site, optimized structural coordinates for NHase species, comparison of experimental and computed $g$ values for $\left[\mathrm{Fe}^{3+}(\mathrm{ADIT})(\mathrm{ADIT}-\mathrm{X})\right]^{+/ 2+}, \mathrm{pH}$ dependence of NHase, and consideration of the assignments of $\mathrm{Na}$ and $\mathrm{N} b$ in light of ENDOR data (PDF)

https://pubs.acs.org/doi/suppl/10.1021/acs.biochem.6b00876/suppl_file/bi6b00876_si_001.pdf

\section{References}

1. Kovacs, J. A. (2004) Chem. Rev. 104, 825- 848 DOI: 10.1021/cr020619e

2. Banerjee, A., Sharma, R., and Banerjee, U. (2002) Appl. Microbiol. Biotechnol. 60, 33- 44 DOI: 10.1007/s00253-002-1062-0

3. Müller, D. and Gabriel, J. (1999) Folia Microbiol. 44, 377- 379 DOI: 10.1007/BF02903708

4. Huang, W., Jia, J., Cummings, J., Nelson, M., Schneider, G., and Lindqvist, Y. (1997) Structure 5, 691- 699 DOI: 10.1016/S0969-2126(97)00223-2

5. Doan, P. E., Nelson, M. J., Jin, H., and Hoffman, B. M. (1996) J. Am. Chem. Soc. 118 (29) 7014- 7015 DOI: 10.1021/ja960611k

6. Yano, T., Ozawa, T., and Masuda, H. (2008) Chem. Lett. 37, 672- 677 DOI: 10.1246/cl.2008.672

7. Dey, A., Chow, M., Taniguchi, K., Lugo-Mas, P., Davin, S., Maeda, M., Kovacs, J. A., Odaka, M., Hodgson, K. O., Hedman, B., and Solomon, E. I. (2006) J. Am. Chem. Soc. 128, 533- 541 DOI: 10.1021/ja0549695 
8. Shearer, J., Callan, P. E., and Amie, J. (2010) Inorg. Chem. 49, 9064- 9077 DOI: 10.1021/ic101765h

9. Dey, A., Chow, M., Taniguchi, K., Lugo-Mas, P., Davin, S., Maeda, M., Kovacs, J. A., Odaka, M., Hodgson, K. O., Hedman, B., and Solomon, E. I. (2006) J. Am. Chem. Soc. 128, 533- 541 DOI: 10.1021/ja0549695

10. Hashimoto, K., Suzuki, H., Taniguchi, K., Noguchi, T., Yohda, M., and Odaka, M. (2008) J. Biol. Chem. 283, 36617- 36623 DOI: 10.1074/jbc.M806577200

11. Brennan, B. A., Alms, G., Nelson, M. J., Durney, L. T., and Scarrow, R. C. (1996) J. Am. Chem. Soc. 118, 9194- 9195 DOI: 10.1021/ja961920d

12. Nelp, M. T., Astashkin, A. V., Breci, L. A., McCarty, R. M., and Bandarian, V. (2014) Biochemistry 53, 3990- 3994 DOI: 10.1021/bi500260j

13. Sugiura, Y., Kuwahara, J., Nagasawa, T., and Yamada, H. (1987) J. Am. Chem. Soc. 109, 5848- 5850 DOI: 10.1021/ja00253a046

14. Kopf, M.-A., Bonnet, D., Artaud, I., Pétré, D., and Mansuy, D. (1996) Eur. J. Biochem. 240, 239244 DOI: 10.1111/j.1432-1033.1996.0239h.x

15. Popescu, V.-C., Münck, E., Fox, B. G., Sanakis, Y., Cummings, J. G., Turner, I. M., and Nelson, M. J. (2001) Biochemistry 40, 7984- 7991 DOI: 10.1021/bi010198f

16. Light, K. M., Yamanaka, Y., Odaka, M., and Solomon, E. I. (2015) Chem. Sci. 6, 6280-6294 DOI: 10.1039/C5SC02012C

17. Rzeznicka, K., Schätzle, S., Böttcher, D., Klein, J., and Bornscheuer, U. T. (2010) Appl. Microbiol. Biotechnol. 85, 1417- 1425 DOI: 10.1007/s00253-009-2153-y

18. Gumataotao, N., Kuhn, M. L., Hajnas, N., and Holz, R. C. (2013) J. Biol. Chem. 288, 1553215536 DOI: 10.1074/jbc.M112.398909

19. Otwinowski, Z. and Minor, W. (1997) Methods Enzymol. 276, 307- 326 DOI: 10.1016/S00766879(97)76066-X

20. Kuhn, M. L., Martinez, S., Gumataotao, N., Bornscheuer, U., Liu, D., and Holz, R. C. (2012) Biochem. Biophys. Res. Commun. 424, 365- 370 DOI: 10.1016/j.bbrc.2012.06.036

21. McCoy, A. J., Grosse-Kunstleve, R. W., Adams, P. D., Winn, M. D., Storoni, L. C., and Read, R. J. (2007) J. Appl. Crystallogr. 40, 658-674 DOI: 10.1107/S0021889807021206

22. Emsley, P., Lohkamp, B., Scott, W. G., and Cowtan, K. (2010) Acta Crystallogr., Sect. D: Biol. Crystallogr. 66, 486- 501 DOI: 10.1107/S0907444910007493

23. Winn, M. D., Ballard, C. C., Cowtan, K. D., Dodson, E. J., Emsley, P., Evans, P. R., Keegan, R. M., Krissinel, E. B., Leslie, A. G., McCoy, A., McNicholas, S. J., Murshudov, G. N., Pannu, N. S., Potterton, E. A., Powell, H. R., Read, R. J., Vagin, A., and Wilson, K. S. (2011) Acta Crystallogr., Sect. D: Biol. Crystallogr. 67, 235- 242 DOI: 10.1107/S0907444910045749

24. Adams, P. D., Afonine, P. V., Bunkóczi, G., Chen, V. B., Davis, I. W., Echols, N., Headd, J. J., Hung, L.-W., Kapral, G. J., Grosse-Kunstleve, R. W., McCoy, A. J., Moriarty, N. W., Oeffner, R., Read, R. J., Richardson, D. C., Richardson, J. S., Terwilliger, T. C., and Zwart, P. H. (2010) Acta Crystallogr., Sect. D: Biol. Crystallogr. 66, 213- 221 DOI: 10.1107/S0907444909052925

25. Hyde, J. S., Bennett, B., Walter, E. D., Millhauser, G. L., Sidabras, J. W., and Antholine, W. E. (2009) Biophys. J. 96, 3354-3362 DOI: 10.1016/j.bpj.2009.01.034

26. Kowalski, J. M. and Bennett, B. (2011) J. Am. Chem. Soc. 133, 1814- 1823 DOI: 10.1021/ja106550u 
27. Stoll, S. and Schweiger, A. (2006) J. Magn. Reson. 178, 42- 55 DOI: 10.1016/j.jmr.2005.08.013

28. Neese, F. (2012) WIREs Comput. Mol. Sci. 2, 73- 78 DOI: 10.1002/wcms.81

29. Nagashima, S., Nakasako, M., Dohmae, N., Tsujimura, M., Takio, K., Odaka, M., Yohda, M., Kamiya, N., and Endo, I. (1998) Nat. Struct. Biol. 5, 347- 351 DOI: 10.1038/nsb0598-347

30. Hopmann, K. H. (2014) Inorg. Chem. 53, 2760- 2762 DOI: 10.1021/ic500091k

31. Adamo, C., Scuseria, G. E., and Barone, V. (1999) J. Chem. Phys. 111, 2889- 2899 DOI: 10.1063/1.479571

32. Perdew, J. P., Burke, K., and Ernzerhof, M. (1996) Phys. Rev. Lett. 77, 3865- 3868 DOI: 10.1103/PhysRevLett.77.3865

33. Fouqueau, A., Mer, S., Casida, M. E., Lawson Daku, L. M., Hauser, A., Mineva, T., and Neese, F. (2004) J. Chem. Phys. 120, 9473- 9486 DOI: 10.1063/1.1710046

34. Schafer, A., Huber, C., and Ahlrichs, R. (1994) J. Chem. Phys. 100, 5829- 5835 DOI: 10.1063/1.467146

35. Schafer, A., Horn, H., and Ahlrichs, R. (1992) J. Chem. Phys. 97, 2571- 2577 DOI: 10.1063/1.463096

36. Weigend, F. and Ahlrichs, R. (2005) Phys. Chem. Chem. Phys. 7, 3297- 3305 DOI: 10.1039/b508541a

37. Stratmann, R. E., Scuseria, G. E., and Frisch, M. J. (1998) J. Chem. Phys. 109, 8218-8224 DOI: $10.1063 / 1.477483$

38. Casida, M. E., Jamorski, C., Casida, K. C., and Salahub, D. R. (1998) J. Chem. Phys. 108, 4439- 4449 DOI: 10.1063/1.475855

39. Bauernschmitt, R. and Ahlrichs, R. (1996) Chem. Phys. Lett. 256, 454- 464 DOI: 10.1016/00092614(96)00440-X

40. Hirata, S. and Head-Gordon, M. (1999) Chem. Phys. Lett. 314, 291- 299 DOI: 10.1016/S00092614(99)01149-5

41. Hirata, S. and Head-Gordon, M. (1999) Chem. Phys. Lett. 302, 375- 382 DOI: 10.1016/S00092614(99)00137-2

42. Neese, F. (2001) J. Chem. Phys. 115, 11080- 11096 DOI: 10.1063/1.1419058

43. Neese, F. (2003) Curr. Opin. Chem. Biol. 7, 125- 135 DOI: 10.1016/S1367-5931(02)00006-6

44. Taylor, C. P. S. (1977) Biochim. Biophys. Acta, Protein Struct. 491, 137-149 DOI: 10.1016/0005-2795(77)90049-6

45. Martinez, S., Wu, R., Sanishvili, R., Liu, D., and Holz, R. (2014) J. Am. Chem. Soc. 136, 11861189 DOI: 10.1021/ja410462j

46. Li, W., Blaesi, E. J., Pecore, M. D., Crowell, J. K., and Pierce, B. S. (2013) Biochemistry 52, 9104- 9119 DOI: 10.1021/bi4010232

47. Jin, H., Turner, I. M., Nelson, M. J., Gurbiel, R. J., Doan, P. E., and Hoffman, B. M. (1993) Coordination Sphere of the Ferric Ion in Nitrile Hydratase J. Am. Chem. Soc. 115, 5290- 5291 DOI: $10.1021 / \mathrm{ja00065a048}$

48. Nelp, M. T., Song, Y., Wysocki, V. H., and Bandarian, V. (2016) A Protein-Derived Oxygen is the Source of the Amide Oxygen of Nitrile Hydratases J. Biol. Chem. 291, 7822- 7829 DOI: 10.1074/jbc.M115.704791 
49. Allison, W. S. and Benitez, L. V. (1972) An Adenosine Triphosphate-Phosphate Exchange Catalyzed by a Soluble Enzyme Couple Inhibited by Uncouplers of Oxidative Phosphorylation Proc. Natl. Acad. Sci. U. S. A. 69, 3004- 3008 DOI: 10.1073/pnas.69.10.3004

50. Kice, J. L. and Cleveland, J. P. (1973) Nucleophilic Substitution Reactions Involving Sulfenic Acids and Sulfenyl Derivatives. the Nucleophile- and Acid-Catalyzed Oxygen-18 Exchange of Phenyl Benzenethiosulfinate J. Am. Chem. Soc. 95, 104- 109 DOI: 10.1021/ja00782a017

51. Lugo-Mas, P., Dey, A., Xu, L., Davin, S. D., Benedict, J., Kaminsky, W., Hodgson, K. O., Hedman, B., Solomon, E. I., and Kovacs, J. A. (2006) How Does Single Oxygen Atom Addition Affect the Properties of an Fe-Nitrile Hydratase Analogue? The Compensatory Role of the Unmodified Thiolate J. Am. Chem. Soc. 128, 11211- 11221 DOI: 10.1021/ja062706k 